\title{
Perfil do idoso em área metropolitana na região sudeste do Brasil: resultados de inquérito domiciliar
}

\section{Profile of the elderly in an metropolitan area of Southeastern Brazil: results of a household survey}

Luiz Roberto Ramos*, Tereza Etsuko da C. Rosa**, Zélia M. Oliveira**, Maria Célia G. Medina**, Francisco R. G. Santos***

\begin{abstract}
RAMOS, L.R. et al. Perfil do idoso em área metropolitana na região sudeste do Brasil: resultados de inquérito domiciliar. Rev. Saúde Pública, 27: 87-94, 1993. Trata-se de estudo multicêntrico visando levantar as necessidades de saúde da população de idosos residentes em zona urbana, conduzido em 6 países na América Latina e coordenado pela Organização Panamericana da Saúde. No Brasil, 1.602 idosos (60 anos e + ) residentes no Distrito de São Paulo, participaram de inquérito domiciliar com questionário de avaliação funcional multidimensional - amostra populacional aleatória, em múltiplos estágios, estratificada por nível socioeconômico. Os resultados mostraram uma populą̧ão bastante carente (70\% tinha uma renda per capita de menos de 100 dólares por mês), vivendo predominantemente em domicilios multigeracionais (59\% viviam com os filhos e/ou com netos), com alta prevalência de doenças crônicas (somente $14 \%$ referiu não ter nenhuma doença) e distúrbios psiquiátricos (27\% foram considerados casos psiquiátricos), e com uma elevada proporção de pessoas com perda de autonomia (47\% precisavam de ajuda para realizar pelo menos uma das atividades da vida diária). Os resultados são analisados tendo em vista as demandas futuras por serviços de saúde especializados e suporte social por parte da crescente população de idosos no Brasil.
\end{abstract}

Descritores: Saúde do idoso. Necessidades e demanda de serviços de saúde. Fatores socioeconômicos.

\section{Introduçáo}

O Brasil, à semelhança dos demais paises latino-americanos, está passando por um processo de envelhecimento populacional rápido $e$ intenso. De $6,3 \%$ da população total, em 1980 , as pessoas com 60 anos ou mais passarão a representar 14\%, em 2.025 - em números absolutos uma das maiores populações de idosos do mundo. As implicaçoes sociais e concernentes à saúde pública são dramáticas e têm sido objeto de discussão (Ramos e col. ${ }^{10}$, 1987; Kalache e col. $\left.{ }^{6}, 1987\right)$.

\footnotetext{
* Setor de Geriatria e Gerontologia da Escola Paulista de Medicina - São Paulo, SP - Brasil.

* Núcleo de Gerontologia do Instituto de Saúde da Secretaria de Estado da Saúde - São Paulo, SP - Brasil.

*** Departamento de Medicina Preventiva da Escola Paulista de Medicina - São Paulo, SP - Brasil
}

Separatas/Reprints: L.R. Ramos - Rua dos Ottonis, 731 - Vila Clementino - 04025-002 - São Paulo, SP . Brasil.

Ediçāo subvencionada pela FAPESP. Processo Medicina 93/ 0208-5
O primeiro inquérito domiciliar visando traçar o perfil do idoso residente em zona urbana no Brasil foi realizado em São Paulo, em 1984. Foi utilizado um instrumento multidimensional - versão brasileira do questionário OARS para avaliação multidimensional do estado funcional, BOMFAQ (Ramos $^{8}, 1987$; Duke University $\left.{ }^{1}, 1978\right)$ - com dados sobre a situação socioeconômica, estrutura familiar, condiçőes de saúde física e mental e grau de autonomia no dia-a-dia dos idosos. Os resultados mostraram que a maioria dos idosos em São Paulo vive em condiçōes de extrema pobreza, fato que pareceu estar associado com a alta frequência de domicílios multigeracionais (convivência do idoso com filhos e netos). Na avaliação do estado de saúde foi observada uma elevada prevalência de doenças crônicas (auto-referidas) e de distúrbios mentais, sendo que mais da metade dos entrevistados referiu necessidade de ajuda parcial ou total para executar as chamadas atividades da vida diária (Ramos, 1987, Ramos e Saad ${ }^{12}$, 1990).

$O$ presente estudo faz parte de uma pesquisa multicêntrica da Organização Panamericana da Saúde realizada em seis países da América Latina; seu objetivo é identificar as necessidades de idosos residentes em zona urbana, através de instru- 
mento de avaliaçð̃o multidimensional com dados sobre utilização de serviços. Paralelamente, foi realizado um inquérito clínico com uma parcela dos entrevistados, no sentido de validar as questões referentes à saúde física.

Serão analisados do presente artigo os dados do inquérito domiciliar, compondo o perfil multidimensional do idoso e comparando-o ao obtido no inquérito anterior. Serão discutidas as implicaçōes do perfil encontrado para o planejamento em saúde.

\section{Metodologia}

Foram selecionados indivíduos com 60 anos ou mais, residentes no Distrito de São Paulo, estratificados pelo nível socioeconômico segundo o subdistrito de residência, de acordo com metodologia anteriormente desenvolvida (Ramos e Goihman" 1989). Como base para a seleção dos subdistritos, foi utilizada a divisão da capital em cinco regiōes homogêneas (Bussab e Dini ${ }^{3}, 1985$ ). De cada região foram escolhidos dois subdistritos de forma a permitir a comparação entre as regiōes: $\mathrm{Bra}$ silândia e Santo Amaro (região 1); Jabaquara e $V$. Guilherme (região 2); Butantã e Tucuruvi (região 3); Aclimação e Santa Cecília (região 4) e Santa Efigênia e Sé (região 5). Os subdistritos grafados foram selecionados com o objetivo adicional de permitir comparaçőes com $o$ inquérito anterior (Ramos $^{8}$, 1987). Posteriormente, o subdistrito da Sé foi excluído do estudo por conter grande número de casas provisórias construídas na época das obras do metrô; no entanto, devido à alta concentração de idosos no subdistrito de Santa Efigênia, a representatividade da região 5 não foi afetada.

\section{Plano Amostral}

A partir dos subdistritos foi realizada uma amostragem em múltiplos estágios, com seleção aleatória de setores censitários e idosos residentes. Os 2.148 setores censitários dos nove subdistritos envolvidos foram ordenados segundo a densidade de idosos fornecida pelo Censo (IBGE5, 1980 ). Cada setor tinha, em média, 60 idosos. Estabeleceu-se que a amostra seria composta de cerca de um terço dos idosos de cada setor. Foram então selecionados aleatoriamente 100 setores na distribuição acumulada de idosos, que seriam suficientes para totalizar cerca de 2.000 idosos. O tamanho da amostra foi proporcional aos recursos disponiveis, dentro de limites aceitáveis para a análise estatística.

Um sistema próprio de referência foi construído pelo arrolamento de todos os idosos dos setores censitários selecionados. Foram listados
8.157 idosos, em média 82 por setor. Considerando-se que a estimativa inicial era de 60 por setor, aplicou-se a seguinte fórmula para corrigir o número de idosos a serem sorteados em cada setor em função do que foi encontrado no arrolamento.

$$
X=20 \times M / N
$$

onde:

$\mathrm{M}=$ número de idosos arrolados no setor

$\mathrm{N}=$ número de idosos no setor pelo Censo $X=$ número de idosos sorteados

O sorteio final dos idosos também foi feito de forma sistemática e aleatória. Calculou-se a fração de expansão $(\mathrm{M} / \mathrm{X})$ e a partir de um número aleatório, como ponto inicial, somou-se a fração de expansão sucessivamente até completar a lista de idosos arrolados no setor. Ao final foram selecionados 2.067 idosos nos 100 setores. Admitindo-se uma rerda amostral de $25 \%$, seriam realizadas cerca de 1.500 entrevistas.

\section{Construção do Instrumento}

O instrumento utilizado foi originalmente concebido pela Organização Panamericana da Saúde (OPS) e posteriormente traduzido para o idioma espanhol e para o português. A versão em espanhol foi aplicada em cinco países latino-americanos Argentina, Chile, Venezuela, Barbados e Trinidade e Tobago. Na versão em português foram acrescentadas algumas questões referentes à condição socioeconômica, saúde mental e presença de doenças crônicas que fizeram parte do questionário BOMFAQ (Ramos ${ }^{8}$, 1987) sem, contudo, comprometer a comparabilidade do questionário com 0 instrumento original aplicado nos demais paises. As perguntas acerca da saúde mental (aspectos emocionais e afetivos) consistiam num screening com 15 questōes que foi previamente validado por entrevista psiquiátrica (Blay e col. $\left.{ }^{2}, 1988\right)$.

\section{Resultados}

Dos 2.007 idosos sorteados, $1.602(80 \%)$ foram efetivamente entrevistados. A percentagem de perda amostral ficou abaixo dos $25 \%$ esperados, na maioria dos subdistritos. As 1.602 entrevistas ficaram assim distribuídas entre os subdistritos: Tucuruvi 393 (84\%); V. Guilherme 87 (90\%); Brasilândia 99 (89\%); Butantã 263 (82\%); Jabaquara 159 (75\%); Santo Amaro 399 (77\%); Santa Cecilia 79 (71\%); Santa Efigênia 48 (79\%) e Aclimação 75 (78\%). Entre parênteses estão indicadas as percentagens das entrevistas realizadas, sob o total sorteado no subdistrito. Do total de entrevistados, apenas 
$43(7,5 \%)$ responderam parcialmente ao questionário, necessitando da ajuda de parentes.

A análise das variáveis demográficas (sexo, idade e origem urbano/rural) e socioeconômicas (renda per capita e nivel de escolaridade) revelou, por um lado, diferenças significativas entre alguns subdistritos (por exemplo: Brasilândia e Aclimação), conforme o esperado pela estratificação socioeconômica. Por outro lado, entre alguns subdistritos de regiōes homogêneas diferentes, como por exemplo Jabaquara e Butantã, as diferenças foram pequenas (Tabela). A partir desta constatação optou-se por reagrupar os subdistritos em três estratos apenas, para efeito de análises futuras, definindo-se estratos de alto, médio e baixo nível socioeconômico, respectivamente denominados de áreas central, intermediária e periférica, e compostas pelos seguintes subdistritos:
Central

- Santa Cecília, Santa Efigênia e Aclimação

Intermediário - Butantã, Tucuruvi, Jabaquara e V. Guilherme

Periférico - Santo Amaro e Brasilândia

\section{Perfil sociodemográfico}

A idade da população entrevistada variou de 60 a 95 anos, com média de 69 anos. Observou-se que a população era, em geral, relativamente jovem, com 58\% abaixo de 70 anos e apenas $10 \%$ acima de 80 anos. Embora sem significância estatística, a área central mostrou uma maior concentração de idosos com idades mais avançadas $14,4 \%$ com 80 anos ou mais (Fig. 1).

Dos 1.602 entrevistados, $40 \%$ eram do sexo masculino e $60 \%$ do feminino. A população femi-

Tabela. Renda per capita e escolaridade dos idosos $(60+)$ residentes em 9 subdistritos do Municipio de São Paulo, 1989.

\begin{tabular}{|c|c|c|c|c|c|}
\hline Subdistrito & \multicolumn{2}{|c|}{ Renda (US\$) } & \multicolumn{2}{|c|}{ Escolaridade } & Estrato \\
\hline $\begin{array}{l}\text { Brasilândia } \\
\text { Santo Amaro } \\
\text { Tucuruvi } \\
\text { V. Guilherme } \\
\text { Butantã } \\
\text { Jabaquara } \\
\text { S. Cecília } \\
\text { S. Efigênia } \\
\text { Aclimação }\end{array}$ & $\begin{array}{l}94 \% \\
79 \% \\
69 \% \\
73 \% \\
54 \% \\
69 \% \\
38 \% \\
50 \% \\
40 \%\end{array}$ & $\begin{array}{r}6 \% \\
21 \% \\
31 \% \\
27 \% \\
46 \% \\
31 \% \\
62 \% \\
50 \% \\
60 \%\end{array}$ & $\begin{array}{r}60 \% \\
44 \% \\
36 \% \\
33 \% \\
36 \% \\
33 \% \\
4 \% \\
13 \% \\
9 \%\end{array}$ & $\begin{array}{r}3 \% \\
15 \% \\
11 \% \\
12 \% \\
6 \% \\
14 \% \\
72 \% \\
28 \% \\
48 \%\end{array}$ & $\begin{array}{l}3 \\
3 \\
2 \\
2 \\
2 \\
2 \\
1 \\
1 \\
1\end{array}$ \\
\hline Total & $70 \%$ & $30 \%$ & $35 \%$ & $18 \%$ & \\
\hline
\end{tabular}

nivel socioeconômico

estrato 1: alto estrato 2: médio

estrato 3: baixo

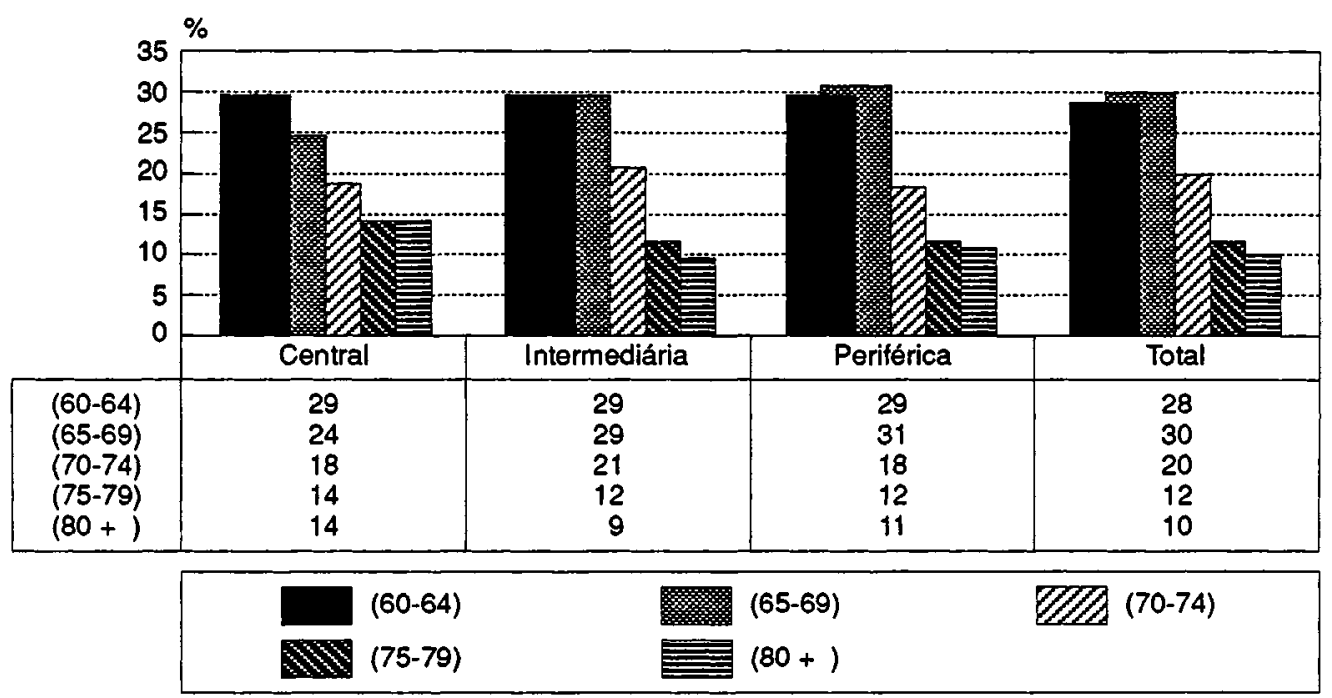

Figura 1. Faixa etária dos idosos $(60+)$ residentes nas áreas central, intermediária e periférica. Município de Sáo Paulo, 1989. 
nina mostrou-se mais densamente concentrada na área central (66\%), enquanto que a masculina mostrou-se mais concentrada na área intermediária (41\%); diferença que, no entanto, não apresentou significância estatística (Fig. 2).

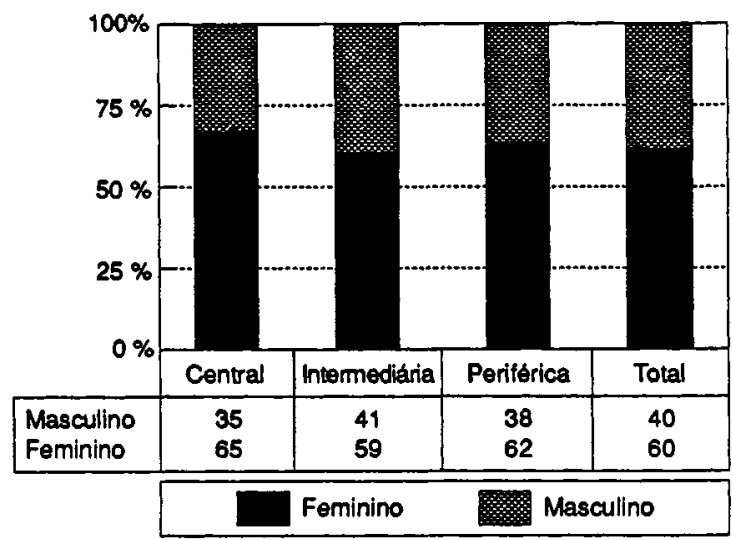

Figura 2. Distribuiçăo por sexo dos idosos $(60+)$ residentes nas áreas central, intermediária e periférica. Municipio de São Paulo, 1989.

A maioria da população entrevistada era oriunda de zona rural (57\%). Chama a atenção o fato de $72 \%$ dos idosos da área periférica serem migrantes da zona rural, enquanto que, na área central, $77 \%$ nasceram em zona urbana (Fig. 3).

Em relação à escolaridade dessa população, encontramos $35 \%$ de analfabetos (foram incluidos nesta categoria as pessoas que responderam saber ler e escrever, mas que nunca freqüentaram escola), $21 \%$ com o nível primário incompleto, $26 \%$

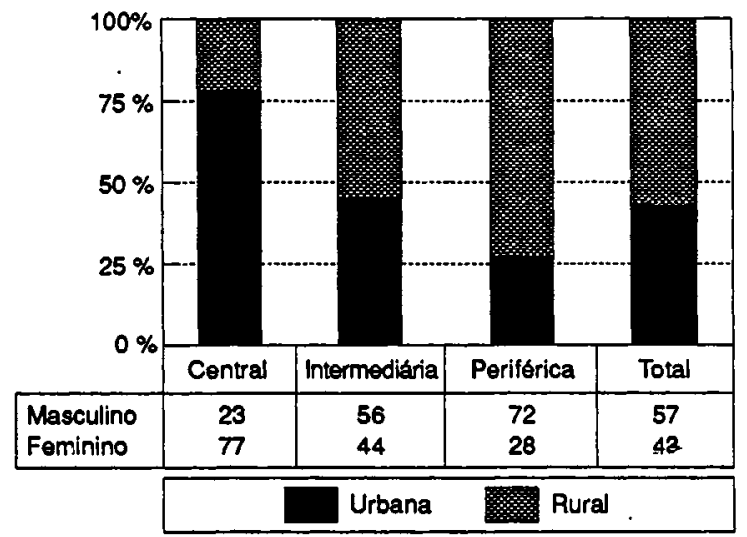

Figura 3. Origem rural/urbana dos idosos $(60+)$ residentes nas áreas central, intermediária e periférica. Municlpio de São Paulo, 1989.

com o primário completo e $18 \%$ que cursaram o pós-elementar (ginasial, colegial e/ou superior). Esses percentuais mudam significativamente em relação às áreas de residência: o índice de analfabetismo aumenta para $46,8 \%$ na periferia, enquanto que e o nível pós-elementar aumenta para 53\% na central (Fig. 4).

A renda per capita mensal do domicílio do idoso era menor que 50 dólares em $39 \%$ dos casos; $32 \%$ tinham uma renda entre 50 e 99 dólares; $22 \%$ entre 100 e 250 dólares; e $7 \%$ ganhavam mais do que 250 dólares. $\mathrm{Na}$ área periférica aumentava significativamente o percentual de idosos com renda per capita mensal menor que $\mathbf{5 0}$ dólares $(56 \%)$ e na área central, em contraste, aumentava a proporção com renda superior a 250 dólares (28\%) (Fig. 5).

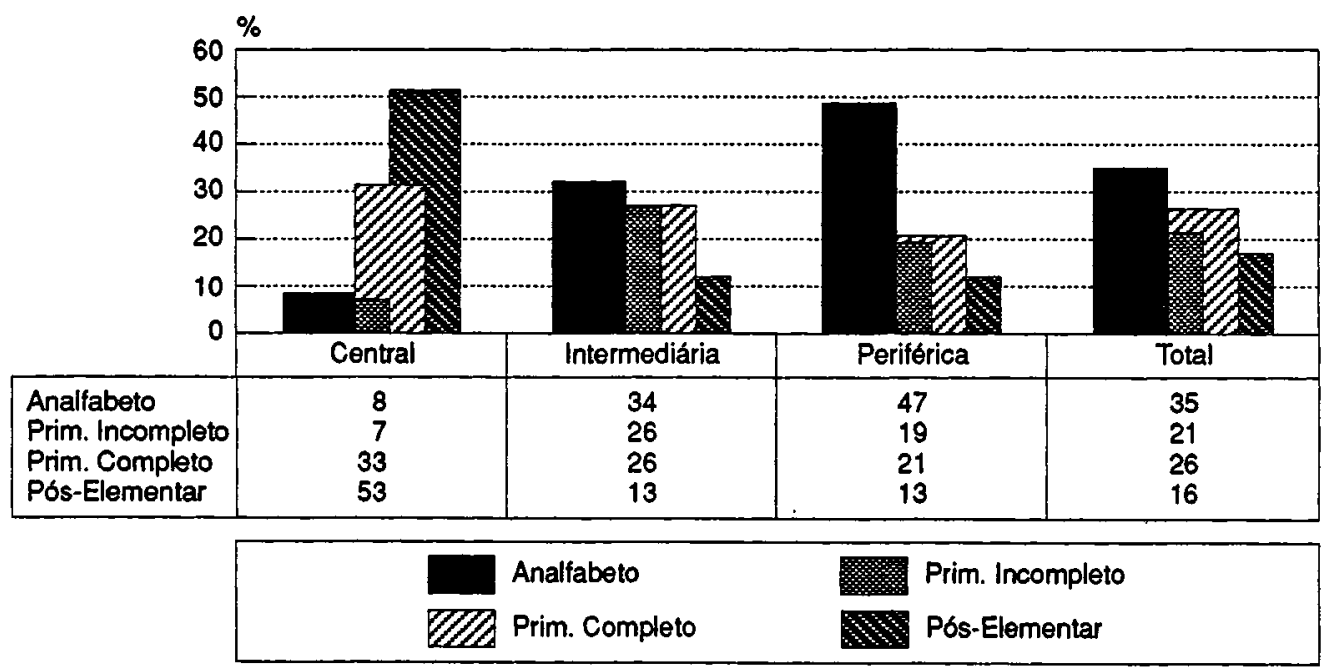

Figura 4. Escolaridade dos idosos $(60+)$ residentes nas áreas central, intermediária e peritérica. Município de Sáo Paulo, 1989. 


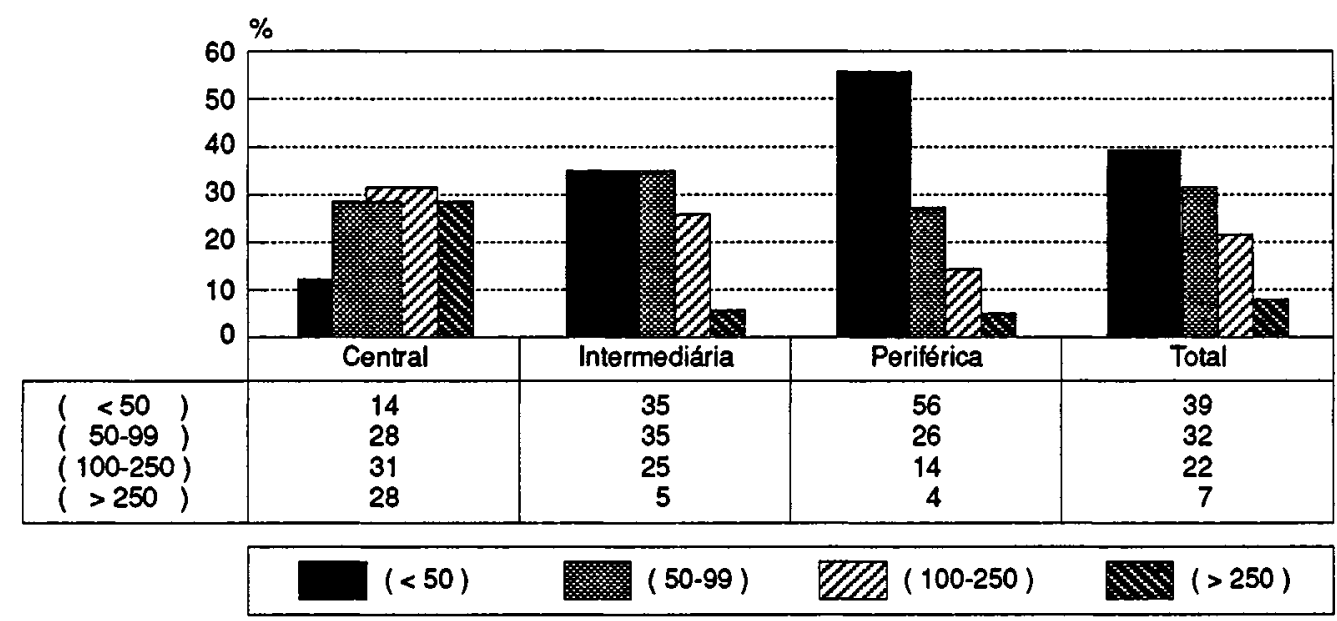

Figura 5. Renda percapita (US $\$$ ) dos idosos $(60+)$ residentes nas áreas central, intermediária e periférica. Municipio de Săo Paulo, 1989.

\section{Perfil multidimensional}

O estudo mostrou que somente uma minoria dos idosos morava só (10\%). A grande maioria vivia em domicílios multigeracionais: coabitavam com esposo(a) e/ou filhos e/ou genros ou noras - domicílio de duas geraçð̃es (34\%); ou ainda com netos - domicilio de três gerações (25\%). Os restantes $31 \%$ moravam apenas com o cônjuge - domicílio de uma geração. Essa distribuição geral variou sensivelmente entre as áreas. Idosos residindo em domicilios de uma geração ou idosos residindo sozinhos predominaram na área central $(41 \%$ e $21 \%$ respectivamente). A convivência em três geraçôes foi mais comum na área periférica (30\%), enquanto que os domicílios de duas geraçōes predominaram na área intermediária (38\%) (Fig. 6).

Em relação ao estado de saúde da população estudada, apenas $14 \%$ consideraram-se livres de doenças crônicas (reumatismo, asma, hipertensão, má circulação, diabetes, derrame, lesões de pele, prisão de ventre e insônia), o que equivale a dizer que $86 \%$ referiram pelo menos uma dessas doenças. Aqueles que têm melhor estado de saúde referida residiam na área central $-21 \%$ responderam não ter nenhuma doença. Os idosos da área periférica foram os que mais responderam positivamente às questōes sobre doenças - $17 \%$ referiram cinco ou mais doenças (Fig. 7).

Mais da metade da população estudada (53\%) referia autonomia total nas atividades da vida diária - preparar refeições, fazer a limpeza da casa, tomar remédios, pentear cabelos, andar no plano, comer, tomar banho, vestir-se, deitar/ levantar da cama, ir ao banheiro em tempo, cortar as unhas dos pés, subir um lance de escada, ir a um lugar necessitando de condução, ir andando a um lugar perto de casa. Para $29 \%$ dos entrevistados era

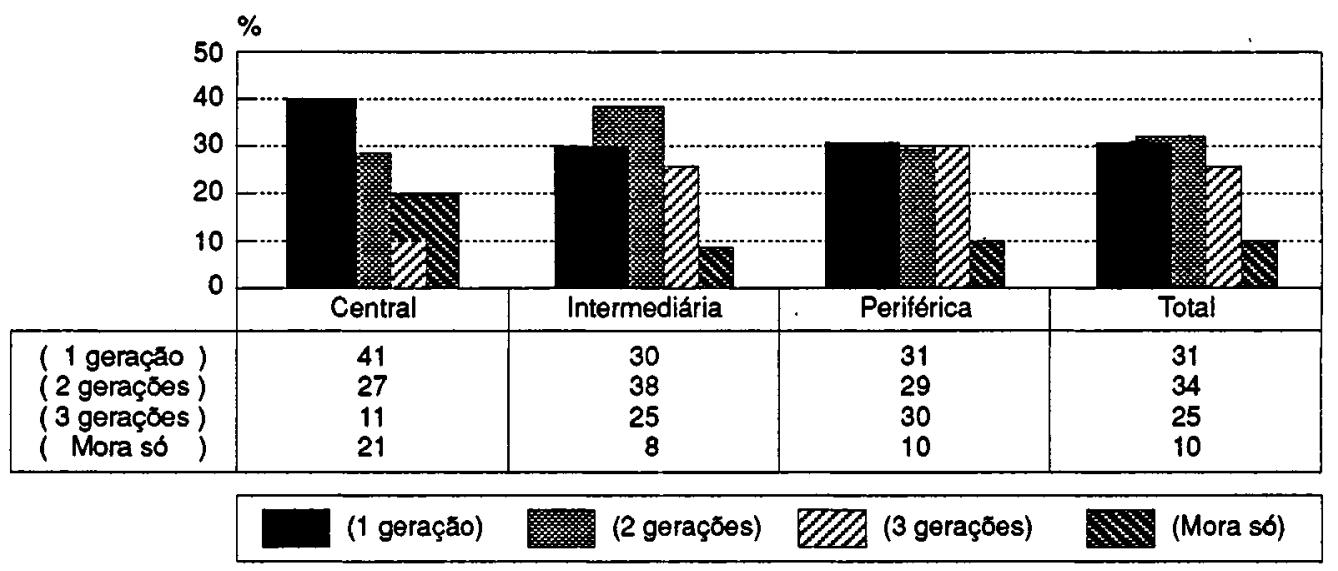

Figura 6. Arranjo domiciliar dos idosos $(60+)$ residentes nas áreas central, intermediária e periférica. Município de Såo Paulo, 1989. 


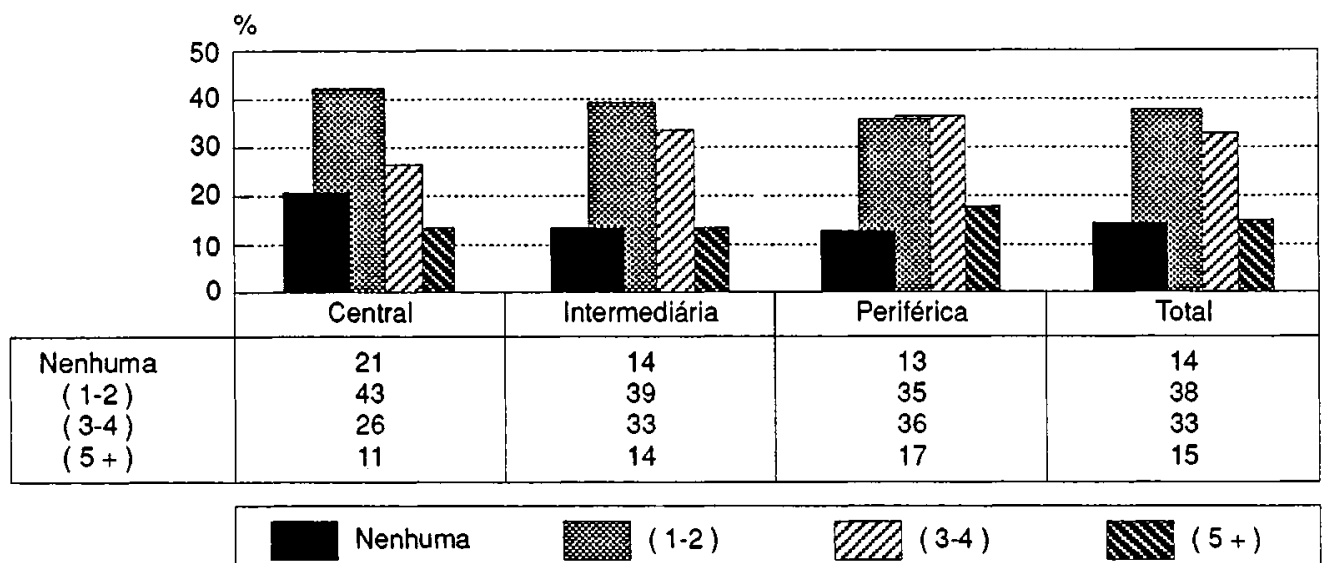

Figura 7. Doenças crônicas referidas pelos idosos residentes nas áreas central, intermediária e periférica. Município de São Paulo, 1989.

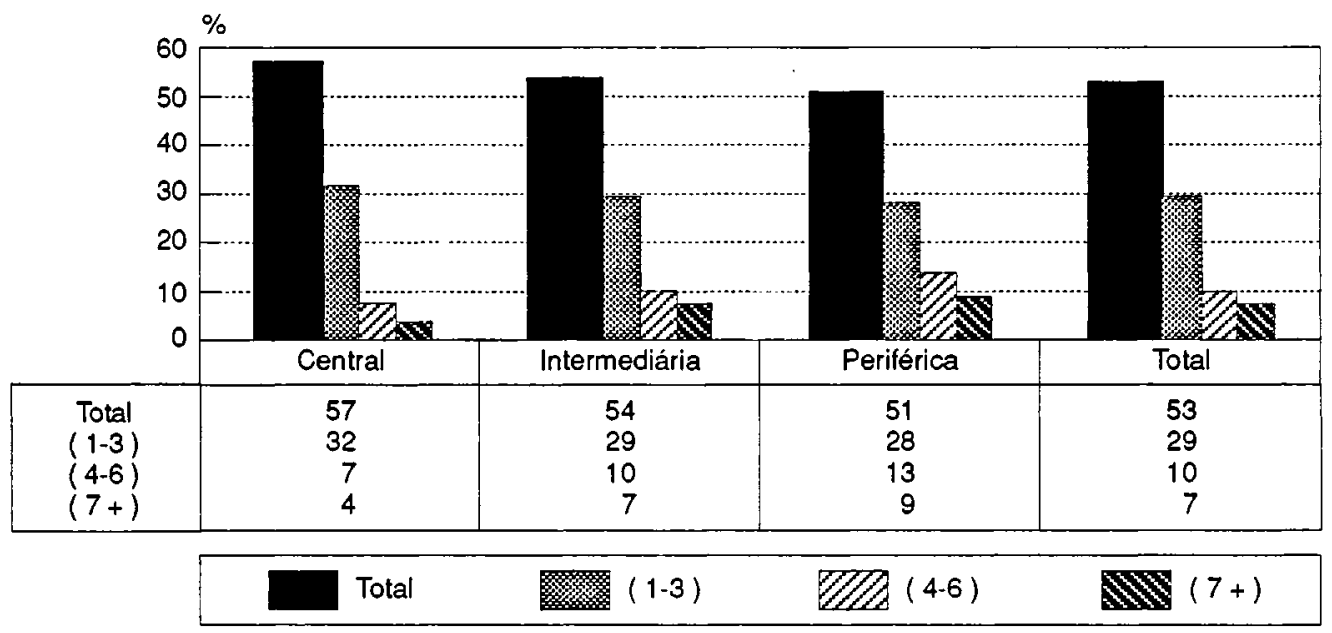

Figura 8. Grau de autonomia (AVD) dos idosos $(60+)$ residentes nas áreas central, intermediária e periférica. Municipio de São Paulo, 1989.

necessária ajuda parcial ou total para realizar até três dessas atividades, e para $10 \%$ era preciso ajuda em mais de três e menos de sete atividades. Para uma minoria considerável $(7 \%)$, foi detectada a necessidade de ajuda parcial ou total para realizar pelo menos sete dessas atividades da vida diária. Considerando-se as áreas geográficas, verificou-se que a autonomia total passa de $51 \%$ na área periférica para $57 \%$ na área central; nesta, apenas $11 \%$ necessitam de ajuda para executar quatro ou mais atividades, enquanto que na periferia a respectiva percentagem dobrou (22\%) (Fig. 8).

A prevalência de respostas positivas - possivelmente "casos" - no screening de saúde mental ( 7 ou mais respostas positivas no total de 15) foi de $27 \%$, percentual que variou significativamente segundo as áreas, sendo maior na periferia (32\%), e bem mais reduzido no centro (18\%) (Fig. 9).

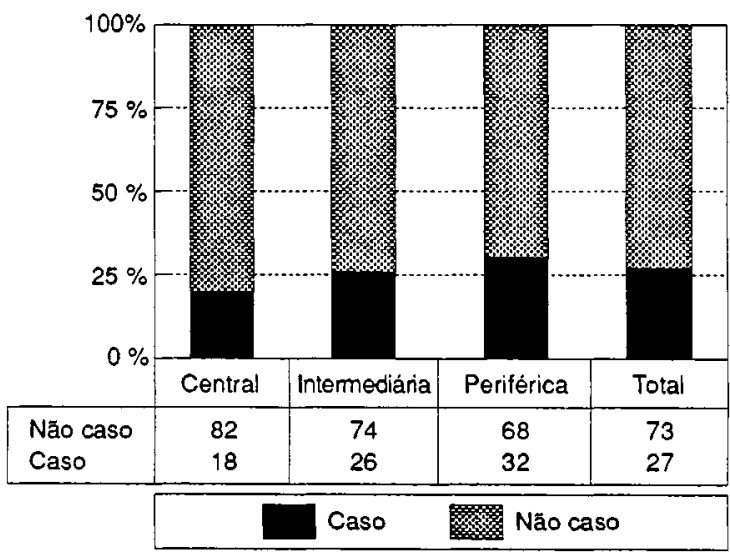

Figura 9. "Screening" de saúde mental positivo entre idosos residentes nas áreas central, intermediária e periférica. Município de São Paulo, 1989. 


\section{Discussão}

O perfil do idoso aqui retratado coincide com o que havia sido previamente descrito por Ramos (1987), com uma amostra menor $(n=303)$, porém com metodologia de amostragem semelhante $e$ instrumento de coleta comparável. Trata-se de população com baixa renda per capita, baixa escolaridade e um passado de migração de zona rural. Essa situação de extrema carência associa-se significativamente com a área periférica definida no processo de amostragem. Nesta região, a população que um dia migrou da zona rural, envelheceu morando em condições precárias, deixando para trás parte da família de criação. A resultante parece ser a tendência observada, qual seja, a de termos o idoso morando no mesmo domicílio que filhos, genros, noras ou netos. Um arranjo domiciliar que se mostra menos freqüente na área central, de nível socioeconômico e educacional mais elevado - o idoso da área central tem uma probabilidade muito maior do que o da periferia de estar morando só, ou em companhia do cônjuge apenas, uma situação que mais se assemelha ao que é discrito, por exemplo, entre idosos de países europeus, embora nestes a proporção de idosos morando sós seja consideravelmente maior (acima de 30\%) (Myers ${ }^{7}, 1992$ ).

Mais de $80 \%$ dos entrevistados referiram pelo menos uma doença crônica; na área periférica $18 \%$ referiu pelo menos cinco doenças como hipertensão, diabetes, problemas vasculares e reumatismo, dentre outras; são afecções que não admitem cura e exigem cuidados freqüentes, prolongados e muitas vezes caros, devido ao uso de técnicas diagnósticas e terapêuticas sofisticadas.

Entre os entrevistados, pouco mais da metade referiu poder realizar todas as atividades do dia-adia sem nenhuma necessidade de ajuda. Quase $20 \%$ precisa de alguma ajuda para realizar pelo menos 4 atividades, e $7 \%$ não realiza sem ajuda pelo menos 7 delas. Este último grupo pode ser considerado como estando num estágio crítico de dependência que, na prática, impossibilita ao indivíduo viver sem uma assistência constante e relativamente especializada. Quanto à saúde mental, 27\% dos idosos apresentavam indícios de comprometimento; na área periférica, a prevalência de distúrbio mental atingia quase um terço dos entrevistados.

A prevalência desses problemas crônicos de saúde com suas incapacidades associadas, não difere grandemente do que se observa em outros países mais desenvolvidos. Trata-se, na verdade, de uma consequiência natural do envelhecimento populacional. O que deve ser objeto de grande preocupação é o fato de esta transição estar se dando num contexto de extrema privação da população; este fato associado ao aumento quase explosivo da população de idosos pode gerar uma demanda insuportável para o sistema de saúde, principalmente no que diz respeito a leitos de longa permanência.

O processo de envelhecimento populacional verificado num centro metropolitano como São Paulo distingue-se, de um lado, por sua rapidez e intensidade e, de outro, pela coexistência com problemas típicos de populações jovens e em franco crescimento. Esse aparente paradoxo faz com que fique ainda mais difícil equacionar uma solução que seja, a um só tempo, factível dentro das limitações de recursos existentes, custobenefício positivo dentro de critérios de saúde pública $\mathrm{e}$, principalmente, adequada às necessidades da população de idosos.

A experiência adquirida na maioria dos países com sistemas de saúde públicos e gratuitos, que vivenciaram o envelhecimento de suas populações, mostra que a tendência é haver um maciça ocupação de leitos hospitalares por idosos (DHSS $\left.{ }^{4}, 1986\right)$. Tendência esta que, nesses países, sobrepuja todos os esforços dos serviços comunitários e domiciliares.

No caso dos domicílios multigeracionais, altamente prevalentes em nosso meio, os dados permitem levantar a hipótese de que antes de ser uma opção cultural, ou humanitária, pode ser um arranjo de sobrevivência, capaz de colocar o idoso numa situação de isolamento e dependência maior do que se ele estivesse morando só (Ramos $\left.{ }^{9}, 1992\right)$.

No entanto, se por um lado o domicílio multigeracional tende a abafar uma demanda por cuidados médicos, sociais, hospitalares, e asilares, por outro lado, esse suporte tende a se colapsar com o aumento do número de idosos dependentes que cada família terá no futuro e com o aumento do grau de incapacidade que cada idoso irá desenvolver, fruto da má qualidade de vida a que estão expostos. Uma família de baixa renda, morando na periferia, e que hoje mora com o idoso, dificilmente poderá continuar nessa situação caso o idoso deixe simplesmente de ser mais um na casa e passe a ser uma pessoa com demandas freqüentes por cuidados médicos e assistenciais. Uma estimativa desse tipo de demanda, que certamente tende a aumentar a cada dia, nos é dada pelos dados, referentes à saúde física, mental e grau de autonomia.

Não existem soluçđ̃es definitivas, dentre todas as já tentadas, por países, em sua maioria, em condições de saúde física e econômica muito melhores do que a nossa. O desafio é grande e o tempo para propor soluçð̃es é curto. Nosso sistema de saúde continua basicamente orientado para o cuidado materno-infantil e o controle das doenças infecciosas. É necessário que se inicie uma reestruturação do sistema no sentido de promover a 
prevenção, diagnóstico precoce e tratamento das doenças crônicas e das incapacidades associadas, no adulto em geral e no idoso em particular.

A filosofia básica deve ser a de procurar manter a autonomia do indivíduo pelo maior tempo possivel, pois com a perda de autonomia, a sobrecarga para a família e para o sistema de saúde tende a se tornar insuportável. Para tanto, é necessário que se institua uma porta de entrada para o idoso no sistema de saúde que não seja o pronto socorro de hospitais de primeira linha, e sim a unidade básica de saúde (UBS), devidamente equipadas com material e pessoal treinado para fazer frente à problemática multiforme do idoso. Dentre as muitas atividades com grande potencial de impacto, na saúde dessa parcela da população, está a fisioterapia, no plano das UBS, com objetivos não só restauradores de incapacidades, mas também preventivos.

Como base para um planejamento a longo prazo, tendo em conta o crescimento da população de idosos, há que se avaliar em maior profundidade o custo-benefício de se instituir a produção em massa de certas cirurgias eletivas que podem mudar drasticamente a autonomia de um idoso - colocação de prótese total de quadril e cirurgia de catarata, por exemplo. Finalmente, é preciso, com urgência, iniciar um planejamento global da rede hospitalar e asilar, já que a demanda por leitos de longa permanência vai, por certo, ser a grande questão colocada para o setor Saúde nesta virada de século.

\section{Agradecimentos}

Organização Panamericana da Saúde na pessoa do Dr. Elias Anzola Pérez, Ministério da Saúde na pessoa do Dr. Renato Maia Guimarães, Dr. José Eduardo C. Moncau, Dra. Cidia Vasconcellos, Marcelo Sampaio Di Pietro, Programa Metropolitano de Saúde do Banco Mundial e CNPq.

RAMOS, L. R. et al. [Profile of the elderly in a metropolitan area of Southeastern Brazil: results of a household survey]. Rev. Saúde Pública, 27: 87-94, 1993. A multicentre study concerned with the health needs of the elderly population living in urban areas and coordinated by the Panamerican Health Organization was undertaken in 6 Latin-American countries. In Brazil, 1,602 elderly residents (of 60 years of age and over) in the District of S. Paulo, constituting a multistage random sample stratified by socioeconomic status, participated in a household survey using a multidemensional functional assessment questionnaire. The results showed a highly deprived population (70\% had a per capita income of less than US\$ 100 per month), living mostly in multi- generational households (59\% were living with children and/or grandchildren), with a high prevalence of chronic physical illnesses (only 14\% referred no illness) and psychiatric disorders $(27 \%$ were considered psychiatric cases), a high proportion of them showing a loss of autonomy (47\% needed help in performing at least one of the activities of daily living). The results are put into perspective concerning future needs in terms of specialized health services and social support for the growing population of elderly people in Brazil.

Keywords: Aging health. Health services needs and demand. Socioeconomic factors.

\section{Referéncias Bibllográficas}

1. DUKE UNIVERSITY CENTER FOR THE STUDY OF AGING AND HUMAN DEVELOPMENT. Multidimensional functional assessment: the OARS methodolo. gy. Durhan, NC, 1978.

2. BLAY, S.; RAMOS, L. R.; MARI, J. J. Validity of a Brazilian version of the Older Americans Resources and Services (OARS) mental health screening questionnaire. $J$. Am. Geriatr. Soc., 36: 687-92, 1988.

3. BUSSAB, W.O. \& DINI, N.P. Pesquisa de emprego e desemprego SEADE/DIEESE: Regiōes homogêneas da Grande São Paulo. Rev. Fund. SEADE, 1(3): 5-11, 1985.

4. DEPARTMENT OF HEALTH AND SOCIAL SECURTTY (DHSS) Hospilal in-patient enquiry: summary tables. London, 1986. OPCS series MB4 $n^{2} 24$, HMSO.

5. FUNDAÇĀO IBGE. Censo demográfico de São Paulo. Rio de Janeiro. 1980. (98 Recenseamento Geral do Brasil). t. 19.

6. KALACHE, A.; VERAS, R. P.; RAMOS, L. R. Envelhecimento da população mundial: um desafio novo. Rev. Saude Pública, 21: 200-10, 1987.

7. MYERS, G. C. Demographic aging and family support for older persons. In: Kendig, H.; Hashimoto, A.; Coppard, L., eds. Family support for the elderly: the international experience. Oxford University Press, New York, 1992. p. 31-68.

8. RAMOS, L. R. Growing old in São Paulo, Brazil: assessment of health status and family support of the elderly of different socio-economic strata living in the community, London, 1987. [PhD Thesis, University of London].

9. RAMOS, L. R. Family support for the elderly in São Paulo, Brazil. In: Kendig, H.; Hashimoto, A.; Coppard, L. eds. Family support for the elderly: the international experience. New York, Oxford University Press, 1992. p. 224-32.

10. RAMOS, L.R.; VERAS, R.P.; KALACHE, A. Envelhecjmento populacional: uma realidade brasileira. Rev. Saúde Pública, 21: 211-24, 1987.

11. RAMOS, L.R. \& GOIHMAN, S. Geographic stratification by socio-economic status: methodology from a household survey with elderly people in São Paulo, Brazil. Rev. Saúde Pública, 23: 478-92, 19897.

12. RAMOS, L.R. \& SAAD, P. Morbidade da população idosa. In: Fundação SEADE. $O$ idoso na Grande Săo Paulo. São Paulo, Fundação Sistema Estadual de Análise de Dados (SEADE), 1990. p. 161-72. (Coleção Realidade Paulista).

Recebido para publicação em 24.6.1992 Reapresentado em 10.2.1993 Aprovado para publicação em 152.1993 